Article

\title{
Effective Delivery of Anti-Cancer Drug Molecules with Shape Transforming Liquid Metal Particles
}

\author{
Dasom Kim, Jangsun Hwang, Yonghyun Choi, Yejin Kwon, Jaehee Jang, Semi Yoon and \\ Jonghoon Choi *
}

School of Integrative Engineering, Chung-Ang University, Seoul 06974, Korea; stvg54@gmail.com (D.K.); isnickawesome@gmail.com (J.H.); dydgus5057@gmail.com (Y.C.); angang1027@gmail.com (Y.K.); jjaeh95@gmail.com (J.J.); semi103306@gmail.com (S.Y.)

* Correspondence: nanomed@cau.ac.kr

Received: 12 September 2019; Accepted: 23 October 2019; Published: 27 October 2019

\begin{abstract}
Liquid metals are being studied intensively because of their potential as a drug delivery system. Eutectic gallium-indium (EGaIn) alloy liquid metals have a low melting point, low toxicity, and excellent tissue permeability. These properties may enable them to be vascular embolic agents that can be deformed by light or heat. In this study, we developed EGaIn particles that can deliver anticancer drugs to tumor cells in vitro and change their shapes in response to external stimuli. These particles were prepared by sonicating a solution containing EGaIn and amphiphilic lipids. The liquid metal (LM)/amphiphilic lipid (DSPC, 1,2-distearoyl-sn-glycero-3-phosphocholin) particles formed a vehicle for doxorubicin, an anticancer drug, which was released (up to 50\%) when the shape of the particles was deformed by light or heat treatment. LM/DSPC particles are non-toxic and LM/DSPC/doxorubicin particles have anticancer effects (resulting in a cell viability of less than 50\%). LM/DSPC/doxorubicin particles were also able to mimic blood vessel embolisms by modifying their shape using precisely controlled light and heat in engineered microchannels. The purpose of this study was to examine the potential of EGaIn materials to treat tumor tissues that cannot be removed by surgery.
\end{abstract}

Keywords: liquid metal; EGaIn; photothermal; drug delivery system; vascular embolism; doxorubicin

\section{Introduction}

The development of new and effective drug carriers to treat cancer is crucial and ongoing [1]. These carriers are usually comprised of biomaterials and are designed to transport small molecules, proteins, DNA, and RNA [2]. When designing drug carriers, aspects such as biocompatibility and biodegradation are carefully considered [3]. Also, they should have low cytotoxicity, and they should be readily absorbed by cells. Drug carriers using metal particles have been widely studied because they are stable, their surface is easy to modify, they are accessible to various drugs, and they are easy to image with MRI or X-ray scanning [4,5].

Metals that are liquid at room temperature are known for their fluidity and conductivity. Mercury, a well-known room temperature liquid metal, is difficult to use in bioresearch because of its toxicity [6]. Alloys such as liquid metals (LM), gallium, gallium-indium common alloys (EGaIn, 75\% Ga, 25\% In), and galinstan (a liquid metal alloy composed from a family of eutectic alloys mainly consisting of gallium, indium, and tin) are promising alternatives to mercury because of their relatively low toxicity $[7,8]$. Gallium-based liquid metals have gained the attention of researchers because they are easily formable, deformable, and stretchable. They are also chemically stable and do not react with water at room temperature [9-11]. In contrast to mercury, unique properties such as high surface tension, good mobility, high electrical conductivity, good biocompatibility, and low toxicity make LM an 
attractive material for biomedical applications, as well as microfluidic systems such as circuits, pumps, electrodes, and sensors [12-16]. In particular, colloids of liquid metals are applied to pumps, sensors, catalysts, and drug delivery systems [17]. LM particles are easily produced by sonication, and can also cause vascular embolisms [18]. They have excellent photochemical and photothermal conversion properties, which can be applied to photothermal conversion agents (PTA) and the photothermal therapy (PTT) of tumors [19-22]. LM particles, however, possess drawbacks that require further research to address. They may cause embolisms in normal tissues; therefore, they must be administered directly to the target vessel using a catheter or stent. Also, their size can be difficult to control after inducing them to change. Furthermore, because no in vivo experiments were performed in this study, a toxicity assessment could not be conducted, and the potential toxicity of the LM particles was not characterized.

Tumor cell proliferation and differentiation are caused by a variety of growth factors, as well as a rich supply of oxygen and nutrients from blood vessels [23]. As tumor growth depends on blood vessels, starving tumors with vascular target therapy is a promising area of study, and research on angiogenesis inhibitors is ongoing [24-26]. However, because tumors become resistant to inhibitors, a new focus of research is on vascular blockage [27]. Several agents that can block blood vessels, including small molecule materials, have been studied along with several physical embolic methods such as coils, balloons, and nanoparticle embolics [28,29].

In this study, engineered liquid metal particles caused in vitro cancer cell necrosis through vascular embolization and conventional drug delivery methods (Figure 1). Using simple sonication techniques, liquid metal particles carrying doxorubicin, an anticancer agent, were formed, and their efficacy was verified. Furthermore, the shape of the liquid metal particles was modified to mimic vascular embolism and verify their potential as a vascular embolizing agent.

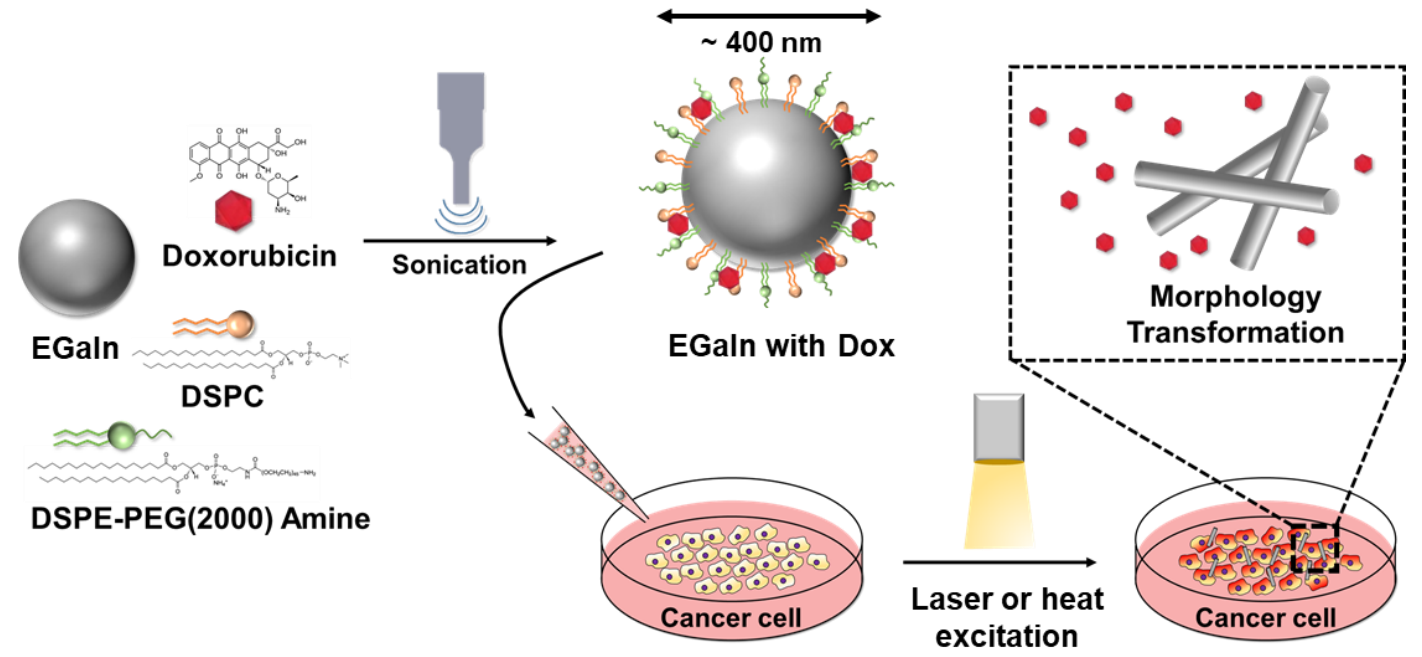

Figure 1. Schematic illustration showing the synthesis of doxorubicin-loaded core-shell liquid metal particles (liquid metal (LM)/1,2-distearoyl-sn-glycero-3-phosphocholin (DSPC)/doxorubicin (DOX)). DSPE-PEG-2000 Amine, 1,2-distearoyl-sn-glycero-3-phosphoethanolamine-N-[amino (polyethylene glycol)-2000]; EGaIn, eutectic gallium-indium.

\section{Materials and Methods}

\subsection{Materials}

All reagents, unless otherwise specified, were purchased from Sigma-Aldrich (St. Louis, MO, USA). 1,2-distearoyl-sn-glycero-3-phosphoethanolamine-N-[amino (polyethylene glycol)-2000] (DSPE-PEG-2000 Amine) and 1,2-distearoyl-sn-glycero-3-phosphocholin (DSPC), which were used to synthesize the particles, were purchased from Avanti Polar Lipids (Alabaster, AL, USA). MDA-MB-231 
breast cancer cells, Hs578T breast cancer cells, and MIA-Paca-2 pancreatic cancer cells were purchased from ATCC (HTB-26, HTB-126, and CRL-1420) (Manassas, VA, USA). A LIVEDEAD ${ }^{\circledR}$ Viability/Cytotoxicity Kit was used to analyze cell viability (Invitrogen, Carlsbad, CA, USA), and a Cell Counting Kit-8 (CCK-8) was obtained from Dojindo (Rockville, MD, USA). A dialysis bag (MW cut-off of 6-8 kDa) was purchased from Spectrumlabs (Piraeus, Greece) to investigate the drug release behavior of the metal particles.

\subsection{Preparation of the LM/DSPC/Doxorubicin (DOX) Particles}

As a general particle synthesis method, we added $10 \mathrm{mg}$ of eutectic gallium-indium (EGaIn) into a $50 \mathrm{~mL}$ disposable sample vial and added $80 \mu \mathrm{L}$ of DSPC ( $25 \mathrm{mg} / \mathrm{mL}$ in chloroform) and $400 \mu \mathrm{L}$ of DSPE-PEG-2000 Amine (25 mg/mL in chloroform) [7,20,30]. We then placed the vial in a bath sonicator (2501E-DTH, BRANSON) and processed it for $10 \mathrm{~min}$ at $50^{\circ} \mathrm{C}$. Next, the chloroform was removed in a dry oven, leaving a powder behind. Then, $10 \mathrm{~mL}$ of deionized (DI) water was added to the powder, mixed, and dispersed using probe sonication (VCX750, SONICS) for one hour with an amplitude of $26 \%$ and a pulse of $5 \mathrm{~s}$ on $/ 5 \mathrm{~s}$ pause. The samples were then centrifuged at $15,000 \times g$ for $10 \mathrm{~min}$, washed twice with $10 \mathrm{~mL}$ of DI water, and left for one hour to remove large particles by gravity. To load the doxorubicin, $4 \mathrm{mg}$ of doxorubicin was dissolved in $4 \mathrm{~mL}$ of DMSO, $2 \mu \mathrm{L}$ of triethanolamine (TEA) was added, and the mixture was incubated at room temperature for $12 \mathrm{~h}$. After the reaction, doxorubicin $(1 \mathrm{mg} / \mathrm{mL})$ was added to the sonicated sample particles so that the ratio of EGaIn and doxorubicin was 10:1 and rocked at $4{ }^{\circ} \mathrm{C}$ for $12 \mathrm{~h}$. After rocking, the samples were centrifuged at $15,000 \times g$ for $10 \mathrm{~min}$ to remove free doxorubicin and rehydrated in $10 \mathrm{~mL}$ of DI water or $1 \times$ Dulbecco's phosphate buffered saline (DPBS, $\mathrm{pH} 7.5)$. The final product was stored at $4{ }^{\circ} \mathrm{C}$.

\subsection{Transmission Electron Microscopy (TEM) and Energy Dispersive X-ray Spectroscopy (EDS)}

To analyze the shape of the synthesized LM/DSPC/DOX particles, the sample was placed on a copper grid and dried. Then, TEM (Talos L120C, FEI, FEI, Hillsboro, OR, USA) analysis was performed at $120 \mathrm{kV}$. Negative staining was not performed owing to the clear brightness difference of the metal particles. EDS imaging and mapping of gallium and indium (JEM-F2000, JEOL) were performed to analyze the composition of the particles.

\subsection{Dynamic Light Scattering (DLS) Analysis}

To determine the size of the particles that contained DSPC and DSPE-PEG-2000-Amine in EGaIn, samples were diluted with water at a ratio of 20:1 and analyzed by dynamic light scattering (DLS) (Zetasizer Nano Zs, Malvern, Malvern Panalytical Ltd., Malvern, UK). The samples were measured six times with a reflective index of 3.9 and an absorption intensity of 0.13 .

\subsection{Ultraviolet and Visible Spectroscopy Analysis}

The absorption wavelength of the LM particles, particles synthesized with DSPC and DSPE-PEG-2000-Amine, and LM particles loaded with doxorubicin was measured with a UV/vis spectrophotometer (BIOMATE 3S, Thermo, Thermo, Waltham, MA, USA). The samples were measured in a $12.5 \times 12.5 \times 45 \mathrm{~mm}$ cuvette at $300 \mathrm{~nm}$ and $900 \mathrm{~nm}$. DI water was used as the blank.

\subsection{DOX-Loading Efficiency and In Vitro Release Test}

To quantitatively analyze the doxorubicin, a standard curve was established using serial dilutions of a stock solution containing $50 \mu \mathrm{g} / \mathrm{mL}$ of doxorubicin in DI water. The fluorescence of each dilution was measured using an excitation wavelength of $480 \mathrm{~nm}$ and an emission wavelength of $560 \mathrm{~nm}$. 
The drug loading efficiency during the synthesis of the LM/DSPC/DOX particles was confirmed by centrifuging the samples for $10 \mathrm{~min}$ at $15,000 \times \mathrm{g}$ and removing the remaining doxorubicin that was not loaded by taking out the supernatant. Using the doxorubicin standard curve as a reference, the fluorescence values of free doxorubicin were analyzed using a multi-plate reader (Synergy H1, BioTek, BioTek, Winooski, VT, USA) at $\lambda$ ex $480 \mathrm{~nm}$ and $\lambda \mathrm{em} 560 \mathrm{~nm}$. The drug-loading efficiency was calculated by measuring the free DOX concentrations. In addition, to confirm the release behavior of the drug from the LM/DSPC/DOX particles, $1 \mathrm{~mL}$ of the sample was placed into a dialysis bag and stored in $5 \mathrm{~mL}$ of $1 \times$ DPBS. Then, $1 \mathrm{~mL}$ of the diffused drug was sampled at various time points during the incubation (e.g., $1 \mathrm{~h}, 2 \mathrm{~h}, 3 \mathrm{~h}, 6 \mathrm{~h}, 9 \mathrm{~h}, 12 \mathrm{~h}, 24 \mathrm{~h}, 48 \mathrm{~h}$, and $72 \mathrm{~h}$ ) at $37^{\circ} \mathrm{C}$ to measure the fluorescence, and $1 \mathrm{~mL}$ of $1 \times$ DPBS was added back to perform an accumulative release.

\subsection{Confocal Laser Scanning Microscopy}

MDA-MB-231 breast cancer cells were used to confirm the behavior, intracellular penetration, and anticancer effects of the LM/DSPC/DOX particles. MDA-MB-231 cells $\left(1.0 \times 10^{5}\right.$ cells/well in an eight-well glass bottom chamber) were incubated in high glucose (Dulbecco's Modified Eagle Medium) DMEM containing 5\% (Fetal Bovine Serum) FBS and 1\% penicillin/streptomycin in a $37^{\circ} \mathrm{C}$ incubator at $5 \% \mathrm{CO}_{2}$. After seeding the cells, they were incubated for one day. LM/DSPC/DOX particles were then added at a concentration of $3 \mu \mathrm{g} / \mathrm{mL}$. The control group was treated with $3 \mu \mathrm{g} / \mathrm{mL}$ of doxorubicin. The samples were incubated for eight hours, washed with $1 \times$ DPBS, and fixed with $4 \%$ paraformaldehyde. The cells were stained with (4', 6 -Diamidine-2' -phenylindole dihydrochloride) DAPI and analyzed using a confocal laser scanning microscope (LSM710, Carl Zeiss, Oberkochen, Germany) at a $\lambda_{\text {ex }}$ of $405 \mathrm{~nm}$ (DAPI) and at a $\lambda_{\text {ex }}$ of $488 \mathrm{~nm}$ (DOX).

\subsection{Live/Dead Assays}

MDA-MB-231 breast cancer cells were used to determine the cell delivery rate and cell death caused by the LM/DSPC/DOX particles. The cells were seeded at a concentration of $5.0 \times 10^{3}$ cells/well in a 96-well culture plate and incubated at $37{ }^{\circ} \mathrm{C}$ with $5 \% \mathrm{CO}_{2}$ for one day. Subsequently, the LM/DSPC/DOX particles were added to the cells at a low $(1 \mu \mathrm{g} / \mathrm{mL})$ and high $(20 \mu \mathrm{g} / \mathrm{mL})$ concentration and incubated for eight hours. After washing with $1 \times$ DPBS, $100 \mu \mathrm{L}$ of each live/dead assay reagent was added, and the cells were incubated at room temperature for $30 \mathrm{~min}$. After the reagent was removed, the cells were washed again with $1 \times$ DPBS and observed using a fluorescence microscope (OX.2053-PLPH, Euromex, Arnhem, The Netherlands).

\subsection{Cytotoxicity Assays}

MDA-MB-231 breast cancer cells, Hs578T breast cancer cells, and MIA-Paca pancreatic cancer cells were used to analyze the time- and concentration-specific cytotoxicity of the LM/DSPC particles. Each cell type was seeded at a concentration of $5 \times 10^{3}$ cells/well in a 96-well culture plate and incubated at $37^{\circ} \mathrm{C}$ with $5 \% \mathrm{CO}_{2}$ for one day. In the hourly cytotoxicity test, MDA-MD-231 cells were treated with control and LM/DSPC/DOX particles using a doxorubicin concentration of $3 \mu \mathrm{g} / \mathrm{mL}$. After washing with $1 \times$ DPBS, the cells were treated with $10 \%$ CCK- 8 reagent and incubated at $37^{\circ} \mathrm{C}$ for two hours. The absorbance was then measured at $450 \mathrm{~nm}$ using a multi plate reader.

\subsection{Light- and Heat-Driven Morphology Changes of LM/DSPC/DOX Particles}

The photothermal/thermal characteristics of the LM/DSPC/DOX particles were observed using laser irradiation or $70{ }^{\circ} \mathrm{C}$ heat treatment. Laser irradiation used a repetition rate of $100 \mathrm{kHz}$, pulse width of $200 \mathrm{~ns}$, scan speed of $100 \mathrm{~mm} / \mathrm{s}$, loop of 10, and power of $2 \mathrm{~W}$. To conduct the heat treatment, the particles were incubated in a dry oven for $30 \mathrm{~min}$ at 50,60 , or $70^{\circ} \mathrm{C}$. Morphological changes were analyzed using TEM according to the experimental conditions outlined above. DLS was used before and after the light/heat treatment to analyze particle sizes according to the particle shape change. 


\subsection{Membrane Blockage Caused by the Shape Transformation of LM/DSPC/DOX Particles}

In order to evaluate the blood vessel embolization potential of LM particles, a membrane perfusion experiment using LM/DSPC particles before and after heat treatment was performed. The membrane was a $40 \mu \mathrm{m}$ pore size nylon cell strainer and the volume of LM/DSPC particles used in this experiment was $1 \mathrm{~mL}$. The absorbance of the particles was measured between $300 \mathrm{~nm}$ and $900 \mathrm{~nm}$ wavelengths before and after passing through the membrane. After the light and temperature treatment $\left(70{ }^{\circ} \mathrm{C}\right.$, $30 \mathrm{~min}$ ), the same measurement was performed. The area of the absorbance range was used to analyze the membrane passage rates altered by the shape variations of particles after the light and temperature treatment.

\subsection{Microfluidic Chip Embolization}

Embolization experiments were carried out using a microfluidic chip introduced to LM/DSPC/DOX particles. A (Polydimethylsiloxane) PDMS chip $20 \mathrm{~mm}$ in length, $10 \mu \mathrm{m}$ in depth, and $10 \mu \mathrm{m}$ in width was fabricated, treated with $\mathrm{O}_{2}$ plasma (FEMTO SCIENCE, Gyeonggi-Do, Korea) at $80 \mathrm{~W}$ for three minutes, and attached to slide glass. Then, $10 \mu \mathrm{L}$ of LM/DSPC/DOX particles at a concentration of $5 \mathrm{mg} / \mathrm{mL}$ were introduced to the microfluidic chip. They then blocked the channel in the chip after heating it to $70^{\circ} \mathrm{C}$ for $30 \mathrm{~min}$. The shape changes and channel blocking behavior of the LM/DSPC/DOX particles were observed using a phase contrast microscope.

\section{Results and Discussion}

\subsection{The Physiological Properties of LM/DSPC/DOX Particles}

The LM/DSPC/DOX particles formed a reddish-gray solution after sonication and were analyzed by TEM. They had a core-shell form of lipids with an EGaIn core (Figure 2a-c). Core-shell particles of various sizes were identified (Supplementary Figure S1). The particles consisted of 75\% gallium, 20\% indium, and $5 \%$ oxygen, which maintained their initial content (Figure $2 \mathrm{~d}, \mathrm{e})$. The average diameter of the particles was $500 \mathrm{~nm}$ with a size distribution ranging from tens to hundreds of nanometers (Figure $2 \mathrm{f}$ and Supplementary Figure S1). Surface analysis using (Field Emission Scanning Electron Microsope) FE-SEM also showed spherical particles and various size distributions (Supplementary Figure S2). When the absorbance of the synthesized particles was measured, they had a very broad absorbance value in all wavelength ranges measured (from UV to near-infrared regions (NIR)) (Figure 2g). At a wavelength of $808 \mathrm{~nm}$, the mass extinction coefficient of the particles was higher than that of gold nanorods, which are often used as photothermal conversion agents [31]. Therefore, an $808 \mathrm{~nm}$ laser was chosen to increase the local temperature and for its biocompatibility in the in vivo light processing experiments. Free doxorubicin not loaded onto the particles was removed to confirm a loading efficiency of approximately $30 \%$. In a drug release test of LM/DSPC/DOX particles loaded with $10 \mu \mathrm{g} / \mathrm{mL}$ of DOX, the drug was released continuously at a rate of up to $60 \%$ discharge over $72 \mathrm{~h}$ (Figure 2h). 

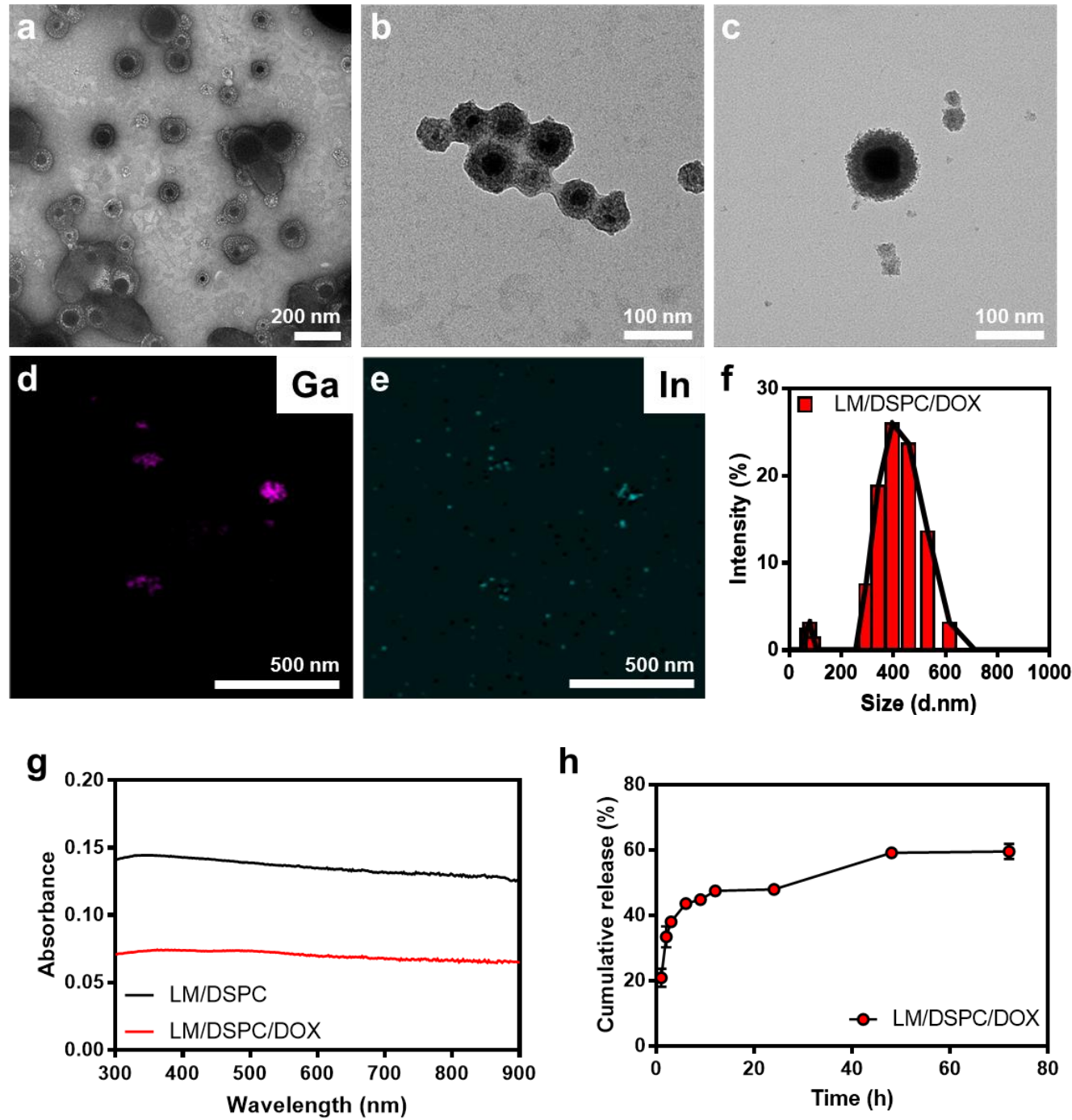

h

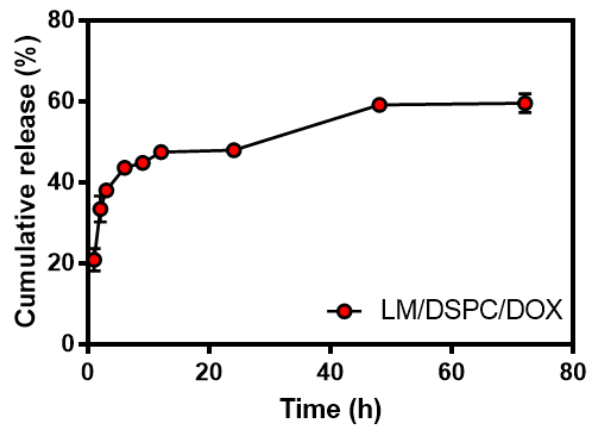

Figure 2. Characteristics of the LM/DSPC/DOX particles. (a-c) Morphology analysis of the LM/DSPC/DOX particles using transmission electron microscopy (TEM) and (d,e) energy dispersive X-ray spectroscopy (EDS) mapping. (f) Size distribution of the LM/DSPC/DOX particles. (g) UV spectroscopy of the LM/DSPC and LM/DSPC/DOX particles. (h) Cumulative doxorubicin release of the LM/DSPC/DOX particles.

\subsection{Cellular Uptake of LM/DSPC/DOX}

After LM/DSPC/DOX particles were introduced to MBA-MD-231 cells, it was confirmed that they were taken up by the cells, the drug molecules were released, and the drug was transported into the nucleus. As a result, the fluorescence intensity appeared higher in the experimental group treated with LM/DSPC/DOX particles when compared with the control group treated with the same concentration $(3 \mu \mathrm{g} / \mathrm{mL})$ of free doxorubicin. The drug penetration into the cells was significant (Figure 3a). Comparing the fluorescence intensity of the cells with image J showed that there was no statistically significant difference between the control group and the experimental group $(n=10$, $p=0.2748$, Figure 3b). However, the enlarged images in Figure 3a show that the drug was delivered to the nucleus of the cell in the experimental group. Larger aggregated particles remain around the cytoplasm of the cell (yellow arrow), suggesting that the smaller particulates effectively migrated into the cell. The particles can be observed around the cytoplasm of the cell even in cells treated with LM/DSPC particles (Supplementary Figure S3a,b). Furthermore, when LM/DSPC/DOX particles were analyzed using confocal microscopy, doxorubicin fluorescence was observed on the particles 
themselves or around the particles, indicating that the drug had, in fact, been loaded onto the particles (Supplementary Figure S3c,d). Thus, it was confirmed that the liquid metal nanoparticles were effectively absorbed by the cancer cells and that doxorubicin was effectively transported into the nucleus of cells within a given time. These data show that LM/DSPC/DOX particles can be used as drug carriers.

a
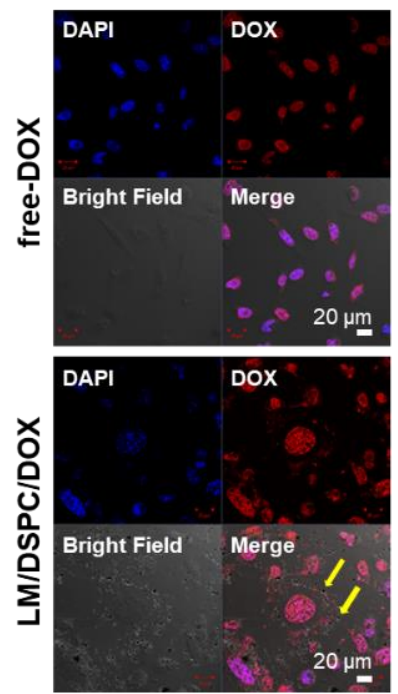

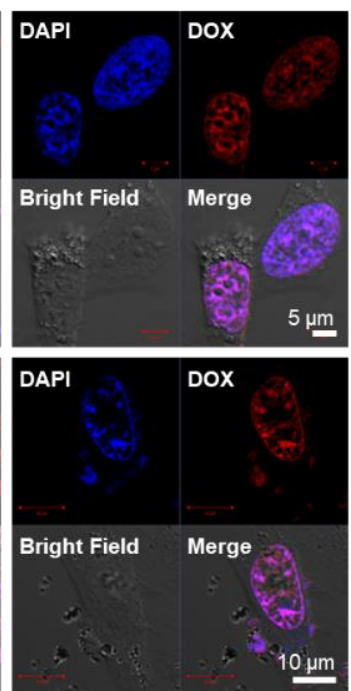

b

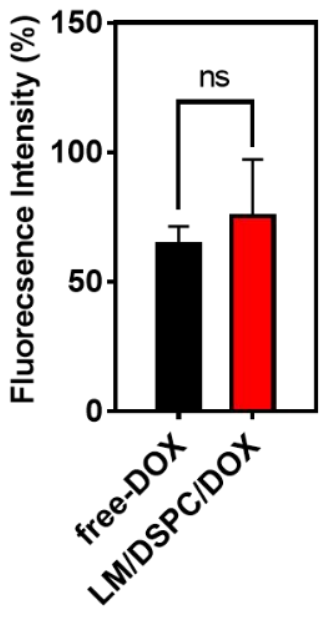

Figure 3. The cellular uptake of doxorubicin $(3 \mu \mathrm{g} / \mathrm{mL})$ and LM/DSPC/DOX particles. (a) Confocal microscopy of MDA-MB-231 breast cancer cells after an $8 \mathrm{~h}$ incubation with doxorubicin (red) and LM/DSPC/DOX particles (red). The nuclei were stained with (4',6-Diamidine-2' -phenylindole dihydrochloride) DAPI (blue). (b) Fluorescence intensity comparison verifying cellular uptake efficacy $(p=0.2748)$. (ns = no significant).

\subsection{The Effect of LM/DSPC/DOX Particle Drug Delivery on Cell Death}

A live/dead assay was used on cancer cells to determine the drug delivery rate of the synthesized LM/DSPC/DOX particles and to determine any anti-cancer effects. MDA-MB-231 breast cancer cells were treated with nanoparticles containing the same concentration of doxorubicin as the control. After eight hours of incubation, the low $(1 \mu \mathrm{g} / \mathrm{mL})$ and high $(20 \mu \mathrm{g} / \mathrm{mL})$ concentrations of DOX-loaded experimental groups showed the same cancer cell killing capacity as the control group treated with free doxorubicin (Figure 4a, Supplementary Figure S4). This demonstrates that doxorubicin was effectively delivered to the cells by the LM/DSPC/DOX particles and that the LM/DSPC/DOX particles are more effective at causing cancer cell necrosis than free doxorubicin.

\subsection{Cell Viability Test Using LM/DSPC and LM/DSPC/DOX Particles}

As most metal particles are toxic, we performed cytotoxicity experiments in cells treated with LM/DSPC particles without DOX loading. LM/DSPC particles did not exhibit cytotoxicity in the three types of cancer cells evaluated (Figure $4 \mathrm{~b}-\mathrm{e}$ ). Several types of cancer cells were treated with LM/DSPC/DOX particles using various concentrations of doxorubicin, and cell viability was analyzed. Particles added to Hs548T cancer cells showed statistically insignificant anti-cancer effects when compared with the cytotoxicity caused by free doxorubicin (Figure $4 b$ ). Treatment of MIA-Paca-2 cells and MDA-MB-231 cells showed similar results (Figure 4c,d). 
a
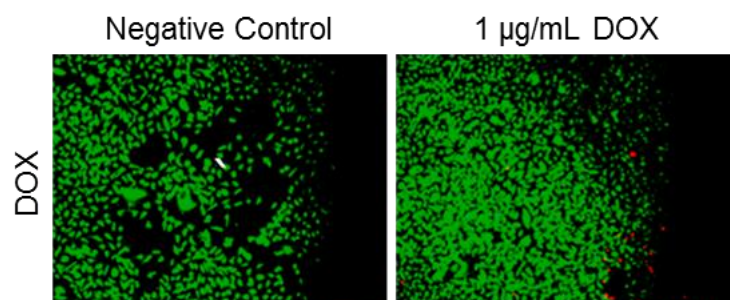

$20 \mu \mathrm{g} / \mathrm{mL}$ DOX

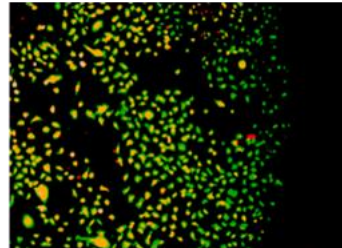

Positive Control
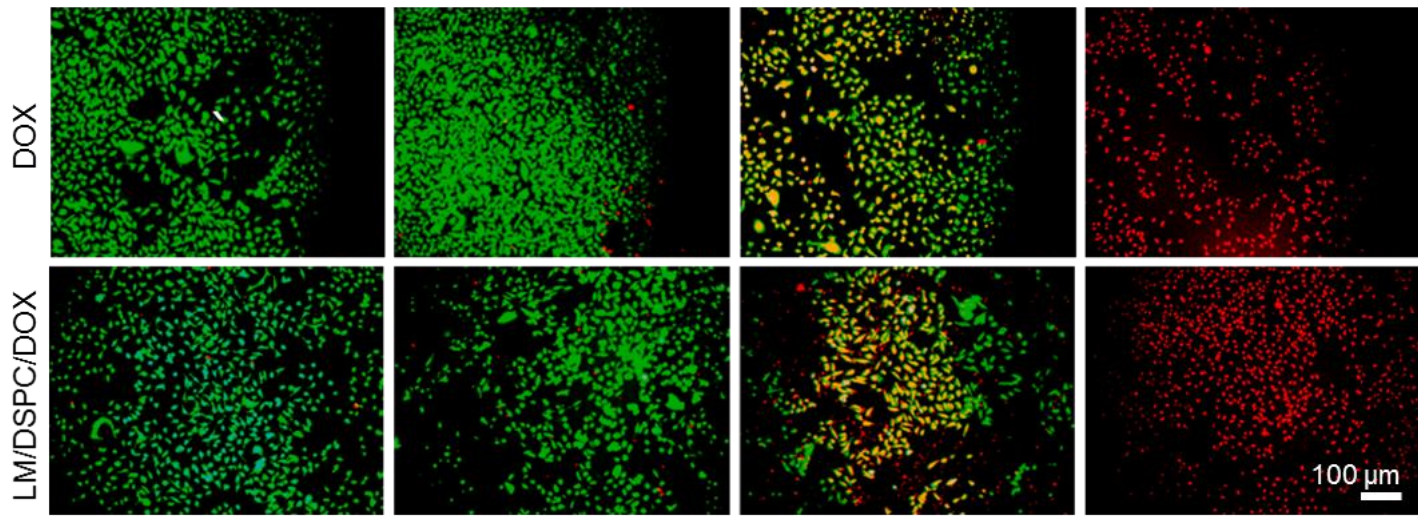

b

Hs578T

C

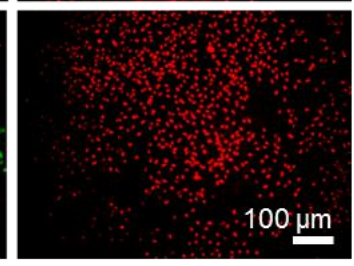

b
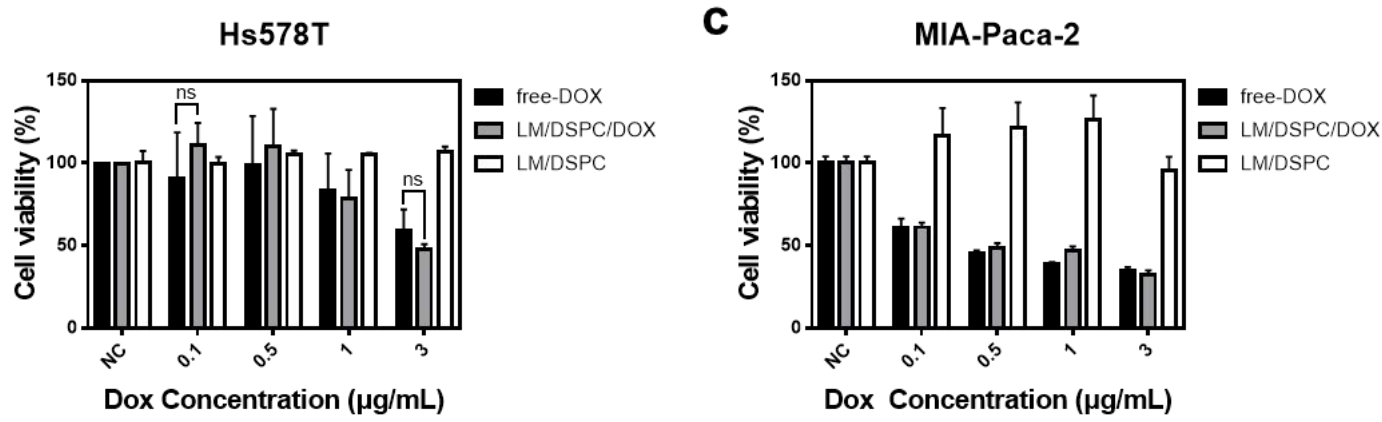

d

MDA-MB-231

e
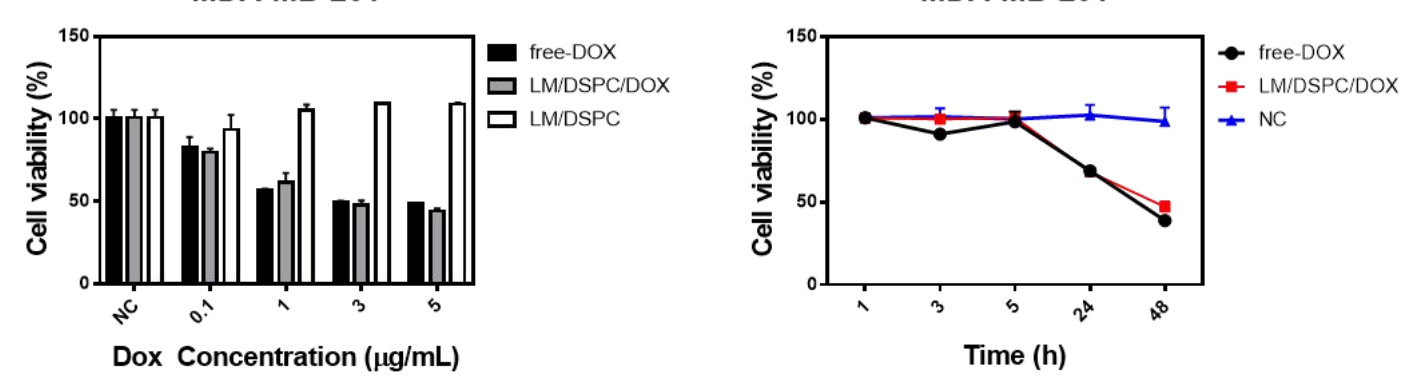

Figure 4. The effect of LM/DSPC/DOX particles on cell viability. (a) A live/dead cell viability assay showing live cells stained with calcein-AM (green) and dead cells stained with EthD-1 (red). (b) Viability of pancreatic cancer cells treated with LM/DSPC/DOX particles loaded with increasing concentrations of doxorubicin. (c,d) Viability of breast cancer cells treated with LM/DSPC/DOX particles loaded with increasing concentrations of doxorubicin. (e) The cytotoxicity of the nanoparticles on MDA-MB-231 cells depends on time ( $\mathrm{NC}=$ negative control).

MDA-MB-231 cancer cells were used to investigate the hourly drug delivery of LM/DSPC/DOX particles. The cell survival rate was similar to that of the control group. After $24 \mathrm{~h}$ of particle and drug treatment, $40 \%$ of the cells died, and after $48 \mathrm{~h}, 60 \%$ of the cells died (Figure $4 \mathrm{e}$ ). These results indicate that LM/DPSC/DOX particles, per se, are not cytotoxic, are efficiently absorbed into cells, and have the same anticancer effects as free DOX in several types of cancer cells.

\subsection{Shape Transition of LM/DSPC Particles Caused by Light and Heat Treatment}

The photothermal characteristics and temperature-dependent changes of the LM/DSPC and LM/DSPD/DOX particles were confirmed by TEM after laser irradiation and heat treatment (Figure 5, 
Supplementary Figure S5). Upon laser treatment, the shape of the two particles changes in a similar fashion, forming rods of around $500 \mathrm{~nm}$ in length (Figure 5a,b). The particles showed a similar tendency upon heat treatment, indicating that their change in shape is due to temperature (Figure $5 \mathrm{c}, \mathrm{d}$ ). In Supplementary Figure S5g-i, the shape change at 50,60, and $70^{\circ} \mathrm{C}$ is shown. However, because it takes a long time to reach a temperature that can cause shape deformation, it will be necessary to design particles that can change their shape more rapidly. In addition, when the size of the particles before and after the heat treatment was measured by DLS and compared, the average diameter of the particles before the heat treatment was $400 \mathrm{~nm}$, but the average particle size after the heat treatment increased to $600 \mathrm{~nm}$. Also, the overall size distribution shifted toward larger diameters (Supplementary Figure S6). Owing to these changes in the shape of LM/DSPC particles, they are a potential vascular embolic material.
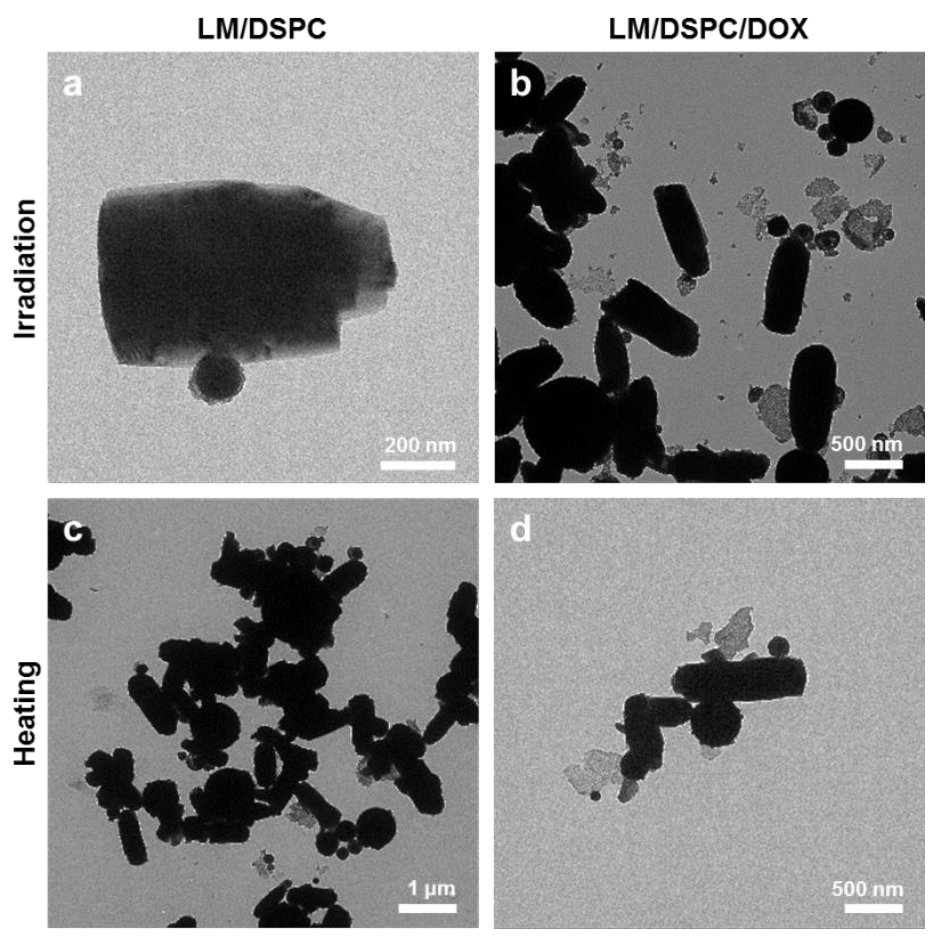

Figure 5. Morphological transformation of LM/DSPC and LM/DSPC/DOX particles. (a,b) TEM images of LM/DSPC and LM/DSPC/DOX particles after laser exposure. (c,d) TEM images of LM/DSPC and $\mathrm{LM} / \mathrm{DSPC} / \mathrm{DOX}$ particles after heating them to $70{ }^{\circ} \mathrm{C}$.

\subsection{Transforming the Shape of LM/DSPC Particles Causes Membrane Occlusion}

To determine whether LM/DSPC particles can cause a vascular embolism, a membrane passage experiment quantified the perfusion of the particles before and after temperature treatment via absorbance with a spectrophotometer (Supplementary Figure S7). The absorbance decreased 49\%, indicating that LM/DSPC particles could pass through the membrane. After applying heat, the LM/DSPC particles agglomerated and failed to cross the membrane because of changes in their size and shape. These data illustrate their potential for blood vessel embolization.

\subsection{Mimicking Vascular Embolization}

Experiments were performed to simulate vascular embolism by flowing LM/DSPC/DOX particles into a microfluidic chip (Figure 6). The particles were dispersed and flowed freely through the channel before heat treatment, but heat treatment caused the particles to change their shape, aggregate, and block the channel (Figure 6c,d). For a more detailed observation, the same experiment was conducted using $10 \mu \mathrm{L}$ of LM/DSPC/DOX particles on a microfluidic chip with a depth of $50 \mu \mathrm{m}$ and a width 
of $100 \mu \mathrm{m}$. At room temperature, the particles gathered via evaporation, but the particles did not block the channel. Upon heat treatment, however, the particles blocked the microchannel in the same way as the microfluidic chip with the smaller sized channels (Supplementary Figure S8). This verifies that LM/DSPC/DOX particles change their shape with heat and, consequentially, may be capable of inducing vascular embolism.
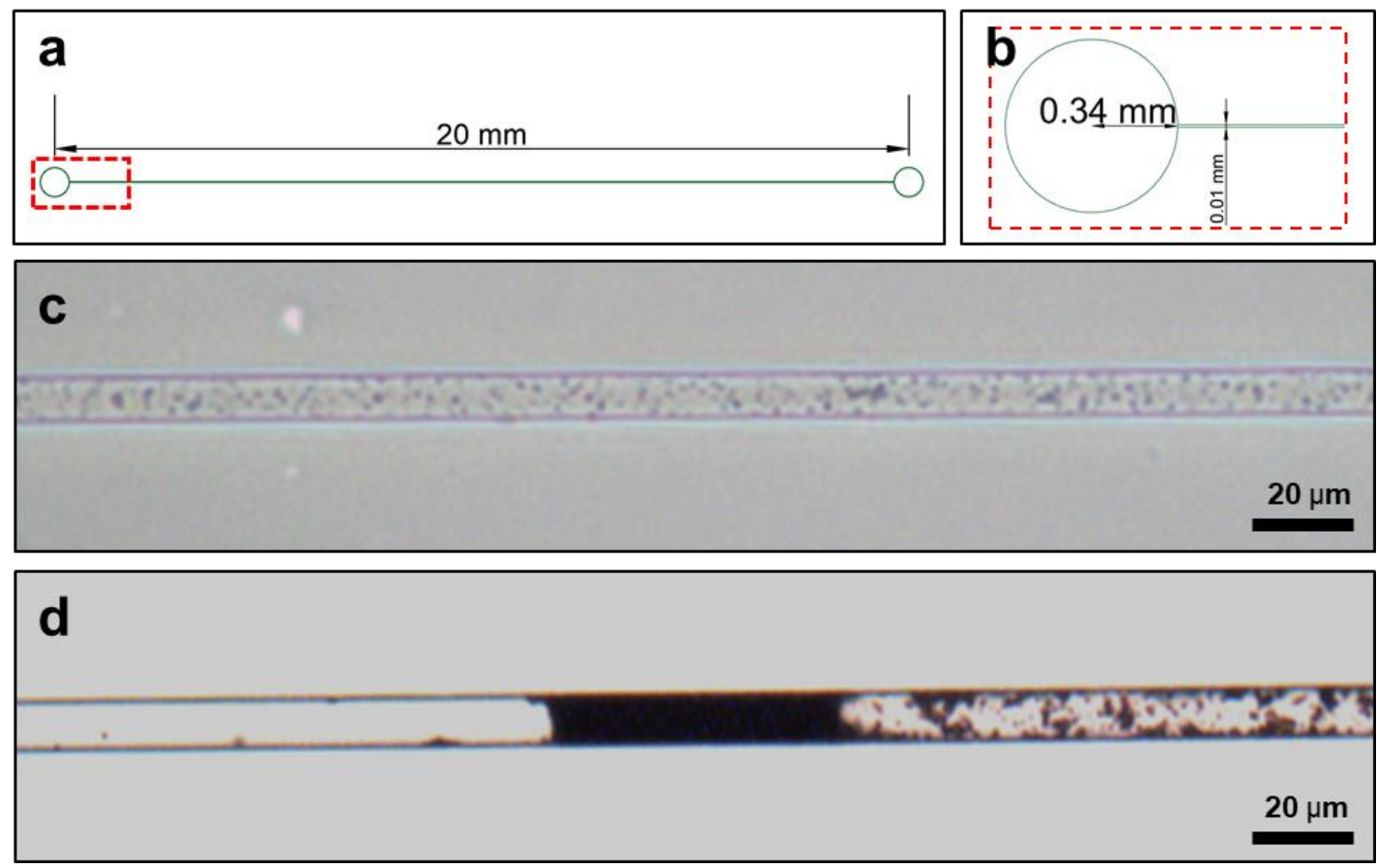

Figure 6. LM/DSPC/DOX particles tested on microfluidic chips as an embolism model. (a,b) The design of the PDMS microfluidic chip. (c) Fluid containing LM/DSPC/DOX particles in a micro channel.

(d) Blocking the micro channel with heat induced transformation of LM/DSPC/DOX particles.

\section{Conclusions}

In this study, we developed a nanoparticle drug carrier and vascular embolic agent that can be applied to tumors that cannot be removed by surgery. The nanoparticles contained an EGaIn core and a lipid DSPC-containing surfactant (DSPE-PEG-2000) containing the anticancer drug doxorubicin. They absorb light at all wavelengths, similar to EGaIn, and have a diameter of approximately $500 \mathrm{~nm}$. This allows them to be treated with infrared light, which is more suitable for in vivo applications. When cancer cells were treated with LM/DSPC/DOX particles, it was confirmed that the particles located to the cell periphery because of their lipid component, and the drug penetrated the cells and was transported to the nucleus. LM/DSPC/DOX particles showed similar anticancer effects to doxorubicin, alone, in several cancer cell types. In particular, the anti-cancer effects of free-dox and LM/DSPC/Dox were the same (approximately 50\%) after $24 \mathrm{~h}$ of Dox ( $3 \mu \mathrm{g} / \mathrm{mL}$ ) treatment. The potential of LM/DSPC/DOX particles as a vascular embolic agent was also demonstrated by their ability to block microfluidic channels after heat treatment. After $10 \mu \mathrm{L}$ of particles $(5 \mathrm{mg} / \mathrm{mL})$ was injected and processed at $70{ }^{\circ} \mathrm{C}$ for $30 \mathrm{~min}$, they successfully blocked the channel flow.

One major limitation of working with LM particles is that changing their shapes in a uniform fashion is difficult to achieve with a laser because it does not evenly transfer heat to the particles. In future experiments, it will be necessary to study methods that add polymers or nanoparticles to LM. Polymers and nanoparticles increase the efficiency of light/heat transfer, which causes LM particles to change their shape. In addition, the drug delivery properties of LM particles should be verified using $3 \mathrm{D}$ cell cultures to ensure the particles can efficiently change their shape at in vivo temperatures. LM 
particles should be tested in future animal experiments as a vascular embolizer for tumor tissues that cannot be treated surgically.

Supplementary Materials: The following are available online at http://www.mdpi.com/2072-6694/11/11/1666/s1, Figure S1: Transmission electron microscopy (TEM) images of LM/DSPC, Figure S2: Field Emission Scanning Electron Microscope (FE-SEM) images of $(a-c)$ LM/DSPC and $(d-f)$ LM/DSPC/DOX, Figure S3: $(a, b)$ Confocal microscopy of MDA-MB-231 breast cancer cell after incubation for $8 \mathrm{~h}$ with LM/DSPC and nuclei (blue). (c,d) Confocal laser scanning microscopy (CLSM) images of LM/DSPC/DOX and doxorubicin (red), Figure S4: Live/Dead cell viability assay. Cells were treated with (a) doxorubicin and (b) LM/DSPC/DOX, Figure S5: TEM images of morphological transformation of LM/DSPC after heating at (a-g) $70{ }^{\circ} \mathrm{C}$, (h) $60{ }^{\circ} \mathrm{C}$ and (i) $50{ }^{\circ} \mathrm{C}$, Figure S6: Dynamic light scattering (DLS) analysis. Size distribution of (a) LM/DSPC/DOX before heating and (b) after heating, Figure S7: Changes in absorbance of LM/DSPC, when LM/DSPC particles passed through the membrane. (a) UV spectroscopy of LM/DSPCs. (b) Membrane passed through the LM/DSPC particles before heating (c) after heating, Figure S8: Blocking of microfluidics channels with LM (channel width $100 \mu \mathrm{m}$ ) (a) Fluid of LM/DSPC/DOX micro/nano particles. (b) Dry the solvent at room temperature. (c) Induce the clogging of channel by heating LM/DSPC/DOX particles.

Author Contributions: D.K., J.H. and J.C. conceptualized the project. D.K., J.H., Y.C., and J.C. designed the experiments. D.K., J.H., Y.K., J.J., and S.Y. executed the experiments. D.K., Y.K., J.J. and SY analyzed the data. D.K., J.H., Y.C. and J.C. wrote the manuscript. J.C. acquired the grants.

Funding: This study was supported by the Nano Material Technology Development Program maintained by the National Research Foundation of Korea (NRF), which is funded by the Ministry of Science and ICT (No. 2017M3A7B8061942) and by the Basic Science Research Program with the help of NRF funds supplied by the Korean government (No. 2019R1A2C1006018).

Conflicts of Interest: The authors declare no conflict of interest.

\section{References}

1. Allen, T.M.; Cullis, P.R.J.S. Drug delivery systems: Entering the mainstream. Science 2004, 303, $1818-1822$. [CrossRef] [PubMed]

2. Langer, R.J.S. New methods of drug delivery. Science 1990, 249, 1527-1533. [CrossRef] [PubMed]

3. Nicolas, J.; Mura, S.; Brambilla, D.; Mackiewicz, N.; Couvreur, P.; Patrick, C. Design, functionalization strategies and biomedical applications of targeted biodegradable/biocompatible polymer-based nanocarriers for drug delivery. Chem. Soc. Rev. 2013, 42, 1147-1235. [CrossRef]

4. Rocca, J.D.; Liu, D.; Lin, W. Nanoscale Metal-Organic Frameworks for Biomedical Imaging and Drug Delivery. Acc. Chem. Res. 2011, 44, 957-968. [CrossRef] [PubMed]

5. Sun, C.-Y.; Qin, C.; Wang, X.-L.; Su, Z.-M. Metal-organic frameworks as potential drug delivery systems. Expert Opin. Drug Deliv. 2013, 10, 89-101. [CrossRef]

6. Clarkson, T.W.; Magos, L. The Toxicology of Mercury and Its Chemical Compounds. Crit. Rev. Toxicol. 2006, 36, 609-662. [CrossRef]

7. Lu, Y.; Hu, Q.; Lin, Y.; Pacardo, D.B.; Wang, C.; Sun, W.; Ligler, F.S.; Dickey, M.D.; Gu, Z. Transformable liquid-metal nanomedicine. Nat. Commun. 2015, 6, 10066. [CrossRef]

8. Dickey, M.D.; Chiechi, R.C.; Larsen, R.J.; Weiss, E.A.; Weitz, D.A.; Whitesides, G.M. Eutectic Gallium-Indium (EGaIn): A Liquid Metal Alloy for the Formation of Stable Structures in Microchannels at Room Temperature. Adv. Funct. Mater. 2008, 18, 1097-1104. [CrossRef]

9. Hohman, J.N.; Kim, M.; Wadsworth, G.A.; Bednar, H.R.; Jiang, J.; LeThai, M.A.; Weiss, P.S. Directing Substrate Morphology via Self-Assembly: Ligand-Mediated Scission of Gallium-Indium Microspheres to the Nanoscale. Nano Lett. 2011, 11, 5104-5110. [CrossRef]

10. Yamaguchi, A.; Mashima, Y.; Iyoda, T. Reversible Size Control of Liquid-Metal Nanoparticles under Ultrasonication. Angew. Chem. Int. Ed. 2015, 54, 12809-12813. [CrossRef]

11. Sheng, L.; Zhang, J.; Liu, J. Diverse Transformations of Liquid Metals Between Different Morphologies. Adv. Mater. 2014, 26, 6036-6042. [CrossRef] [PubMed]

12. Tang, S.Y.; Khoshmanesh, K.; Sivan, V.; Petersen, P.; O’Mullane, A.P.; Abbott, D.; Kalantar-zadeh, K. Liquid metal enabled pump. Proc. Natl. Acad. Sci. USA 2014, 111, 3304-3309. [CrossRef] [PubMed]

13. Zhang, J.; Yao, Y.; Sheng, L.; Liu, J. Self-Fueled Biomimetic Liquid Metal Mollusk. Adv. Mater. 2015, 27, 2648-2655. [CrossRef] [PubMed] 
14. Boley, J.W.; White, E.L.; Chiu, G.T.-C.; Kramer, R.K. Direct Writing of Gallium-Indium Alloy for Stretchable Electronics. Adv. Funct. Mater. 2014, 24, 3501-3507. [CrossRef]

15. So, J.-H.; Dickey, M.D. Inherently aligned microfluidic electrodes composed of liquid metal. Lab Chip 2011, 11,905-911. [CrossRef]

16. Liu, T.; Sen, P.; Kim, C.-J. Characterization of Nontoxic Liquid-Metal Alloy Galinstan for Applications in Microdevices. J. Microelectromech. Syst. 2011, 21, 443-450. [CrossRef]

17. Gao, M.; Gui, L. A handy liquid metal based electroosmotic flow pump. Lab Chip 2014, 14, 1866-1872. [CrossRef]

18. Wang, Q.; Yu, Y.; Liu, J. Delivery of Liquid Metal to the Target Vessels as Vascular Embolic Agent to Starve Diseased Tissues or Tumors to Death. 2014. Available online: https://arxiv.org/ftp/arxiv/papers/1408/1408. 0989.pdf (accessed on 4 August 2014).

19. Yi, L.; Liu, J. Liquid metal biomaterials: A newly emerging area to tackle modern biomedical challenges. Int. Mater. Rev. 2017, 62, 415-440. [CrossRef]

20. Lu, Y.; Lin, Y.; Chen, Z.; Hu, Q.; Liu, Y.; Yu, S.; Gao, W.; Dickey, M.D.; Gu, Z. Enhanced Endosomal Escape by Light-Fueled Liquid-Metal Transformer. Nano Lett. 2017, 17, 2138-2145. [CrossRef]

21. Wang, X.; Yao, W.; Guo, R.; Yang, X.; Tang, J.; Zhang, J.; Gao, W.; Timchenko, V.; Liu, J. Soft and Moldable Mg-Doped Liquid Metal for Conformable Skin Tumor Photothermal Therapy. Adv. Health Mater. 2018, 7, 1800318. [CrossRef]

22. Sun, X.; Sun, M.; Liu, M.; Yuan, B.; Gao, W.; Rao, W.; Liu, J. Shape tunable gallium nanorods mediated tumor enhanced ablation through near-infrared photothermal therapy. Nanoscale 2019, 11, 2655-2667. [CrossRef] [PubMed]

23. Witsch, E.; Sela, M.; Yarden, Y. Roles for growth factors in cancer progression. Physiology 2010, $25,85-101$. [CrossRef]

24. Folkman, J. Advances in Cancer Research; Elsevier: Amsterdam, The Netherlands, 1974; Volume 19331-19358.

25. Jain, R.K. Vascular and interstitial barriers to delivery of therapeutic agents in tumors. Cancer Metastasis Rev. 1990, 9, 253-266. [CrossRef] [PubMed]

26. Thorpe, P.E. Vascular targeting agents as cancer therapeutics. Clin. Cancer Res. 2004, 10, 415-427. [CrossRef]

27. Thorpe, P.E.; Chaplin, D.J.; Blakey, D.C. The first international conference on vascular targeting: meeting overview. Cancer Res. 2003, 63, 1144-1147. [PubMed]

28. Denekamp, J. Commentary: The tumour microcirculation as a target in cancer therapy: A clearer perspective. Eur. J. Clin. Investig. 1999, 29, 733-736. [CrossRef]

29. Hori, K.; Saito, S.; Kubota, K. A novel combretastatin A-4 derivative, AC7700, strongly stanches tumour blood flow and inhibits growth of tumours developing in various tissues and organs. Br. J. Cancer 2002, 86, 1604-1614. [CrossRef]

30. Chechetka, S.A.; Yu, Y.; Zhen, X.; Pramanik, M.; Pu, K.; Miyako, E. Light-driven liquid metal nanotransformers for biomedical theranostics. Nat. Commun. 2017, 8, 15432. [CrossRef]

31. Hu, J.J.; Liu, M.D.; Chen, Y.; Gao, F.; Peng, S.Y.; Xie, B.R.; Zhang, X.Z. Immobilized liquid metal nanoparticles with improved stability and photothermal performance for combinational therapy of tumor. Biomaterials 2019, 207, 76-88. [CrossRef]

(C) 2019 by the authors. Licensee MDPI, Basel, Switzerland. This article is an open access article distributed under the terms and conditions of the Creative Commons Attribution (CC BY) license (http://creativecommons.org/licenses/by/4.0/). 